\title{
Semi-relativistic charge-current density operator
}

\author{
S. Boffi, F. Capuzzi, P. Demetriou and M. Radici \\ Dipartimento di Fisica Nucleare e Teorica, Università di Pavia, and \\ Istituto Nazionale di Fisica Nucleare, Sezione di Pavia, Pavia, Italy
}

\begin{abstract}
The charge-current density and two-photon operators consistent with a single-particle semi-relativistic Hamiltonian are derived within a suitable functional derivative formalism which preserves gauge invariance. An application to electron scattering is presented and results are compared with a fully relativistic case and the non-relativistic cases corrected through fourth order in $M^{-1}$.
\end{abstract}




\section{Introduction}

The structure and dynamics of strongly interacting systems, such as nuclei and nucleons, is best studied using an electromagnetic probe. By treating the electromagnetic interaction within quantum electrodynamics theoretical uncertainties are minimized and the relevant information is simply related to charge- and current-density distributions (for a recent review, see [1]). However, ambiguities may arise due to an inconsistent treatment of the different theoretical ingredients belonging to the complex description of the underlying many-body problem.

In particular, in connection with relativity one meets two orders of problems. On the one hand, the elementary operator is well known. To be specific, let us start considering a free spin- $\frac{1}{2}$ particle with mass $M$. In terms of normalized spinors, the matrix element of the four-current describing its transition from an initial state $|\vec{p}, s\rangle$ (with spinor $u_{i}$ and total energy $E_{\vec{p}}=\sqrt{|\vec{p}|^{2}+M^{2}}$ ) to a final state $\left|\vec{p}^{\prime}, s^{\prime}\right\rangle$ (with spinor $u_{f}$ and total energy $\left.E_{\vec{p}^{\prime}}=\sqrt{\left|\overrightarrow{p^{\prime}}\right|^{2}+M^{2}}\right)$ is written as

$$
\left\langle\vec{p}^{\prime}, s^{\prime}\left|j^{\mu}\right| \vec{p}, s\right\rangle=\frac{M}{\sqrt{E_{\vec{p}} E_{\vec{p}^{\prime}}}} \bar{u}_{\mathrm{f}}\left[\gamma^{\mu} F_{1}\left(Q^{2}\right)+\mathrm{i} \sigma^{\mu \nu} q_{\nu} \frac{\kappa}{2 M} F_{2}\left(Q^{2}\right)\right] u_{\mathrm{i}}(2 \pi)^{-3} \mathrm{e}^{-\mathrm{i} \vec{q} \cdot \vec{x}}
$$

where $Q^{2}=-q_{\nu} q^{\nu}=\vec{q}^{2}-\omega^{2}$ is defined in terms of the energy $\omega$ and momentum $\vec{q}$ of the absorbed photon $\left(\vec{q}=\vec{p}^{\prime}-\vec{p}\right) ; F_{1}$ and $F_{2}$ are the Dirac and Pauli form factors, respectively, and $\kappa$ is set equal to the anomalous part of the magnetic moment of the particle. Alternatively, making use of the Gordon decomposition one writes

$$
\left\langle\vec{p}^{\prime}, s^{\prime}\left|j^{\mu}\right| \vec{p}, s\right\rangle=\frac{M}{\sqrt{E_{\vec{p}} E_{\vec{p}^{\prime}}}} \bar{u}_{\mathrm{f}}\left[\left(p^{\mu}+p^{\prime \mu}\right) \Gamma_{1}\left(Q^{2}\right)+\gamma^{\mu} \Gamma_{3}\left(Q^{2}\right)\right] u_{\mathrm{i}}(2 \pi)^{-3} \mathrm{e}^{-\mathrm{i} \vec{q} \cdot \vec{x}}
$$

where

$$
F_{1}\left(Q^{2}\right)=2 M \Gamma_{1}\left(Q^{2}\right)+\Gamma_{3}\left(Q^{2}\right), \quad \kappa F_{2}\left(Q^{2}\right)=-2 M \Gamma_{1}\left(Q^{2}\right) .
$$

The current of a composite system is often calculated in the impulse approximation, i.e. under the assumption that it is given by the sum of the 
currents of the individual constituents, treated as free particles. Under this assumption, the system current results from the sum of terms like one of the above currents. However, the use of a relativistic expression for the current inside matrix elements requires that the corresponding states are also treated relativistically. This is not always possible in many-body theories of strongly interacting systems where most information on the structure of nuclei and baryons comes from solving the non-relativistic Schrödinger equation. Therefore, one is faced with the problem of a non-relativistic reduction of the charge-current density operator.

There are two ways to obtain a non-relativistic approximation including lowest-order relativistic corrections, when constructing an effective Hamiltonian which is to be used in non-relativistic perturbation theory [2]. In the first, one evaluates the matrix element of the potential operator between positive energy solutions of the free Dirac equation and then performs a twocomponent reduction (direct Pauli reduction). Low-energy theorems and gauge invariance are satisfied at the price, e.g., that the electromagnetic interaction of quarks contains a non additive (two-body) term and the effects of the quark binding potential [3].

The second way is the Foldy-Wouthuysen (FW) method [4]. It is shown that the two methods are equivalent to first order in perturbation theory, but differ to higher order. Moreover, only the FW effective $S$-matrix reproduces the full relativistic $S$-matrix [2].

The FW method looks for a canonical transformation which decouples the Dirac equation into two two-component equations, one reducing to the Pauli description in the non-relativistic limit, the other describing negative-energy states. Relativistic corrections can be classified according to the expansion in powers of a suitable parameter. A natural scale can be identified with $Q / 2 M$. The formal series expression of the transformed Hamiltonian must be truncated at some order which will correspond to a non-relativistic expansion of the transformed Hamiltonian in a power series in $M^{-1}$. This was done through second order by McVoy and Van Hove [5] and through fourth order by Giusti and Pacati [6]. However, in electromagnetic interactions with nuclei a new scale emerges essentially related to the large anomalous magnetic moment $\kappa$ of the nucleon. Therefore, $\kappa Q / 2 M$ must also be considered as a suitable expansion parameter when extending the expansion to larger momenta [7].

The FW method has been successfully applied to inclusive and semi- 
inclusive scattering of electron by nuclei [1], but may easily break down when increasing the energy $\omega$ and momentum $\vec{q}$ transferred by the photon.

On the other hand, the non-relativistic description of the many-body system itself may become too crude an approximation when one is exploring extreme conditions in nuclei or simply considers that in quark models relativistic effects are most important because the mean velocity of a constituent quark in the nucleon is comparable with the velocity of light. The relativistic dynamics of interacting particles is nontrivial and two different approaches have been used to investigate relativistic effects in binding energies. For nuclei the theory is patterned after quantum electrodynamics and is relativistically covariant (for a review see [8]). In this approach the antinucleon degrees of freedom play an important role. However, there is no clear evidence of a simple coupling of $N \bar{N}$ states to mesons and photons. In the other approach, starting from a relativistically covariant description of a system of interacting particles where the interactions are direct rather than mediated through a field [9, 10, 11, 12], not manifestly covariant but relativistic Hamiltonians have been proposed to study both hadrons [13, 14, 15, 16, 17] and nuclei [18, 19, 20, 21]. In all cases the kinetic energy operator was taken as

$$
T=\sqrt[+]{\vec{p}^{2}+M^{2}}
$$

By this choice one excludes negative energy states ab initio and simply solves a Schrödinger equation for bound states. Relativized models have been developed accordingly and also applied. e.g., to investigate electroexcitation of baryon resonances [22, 23, 24, 25].

The problem of finding a charge-current density operator consistent with a single-particle semi-relativistic Hamiltonian, i.e. a Hamiltonian consisting of a kinetic energy given by eq. (4) and of an external, energy and momentum independent potential, is addressed in sect. 3. The derivation makes use of the functional derivative formalism developed in sect. 2 to ensure gauge invariance (see also [26]).

In this paper we will not consider the fact that hadrons are bound in the many-body system and a proper modification of the current is necessary in order to account for off-shell effects that can only be specified when a sufficient knowledge of the internal dynamics is available. A variety of prescriptions have been proposed to solve this problem in specific cases (see, e.g., [27, 28, 29, 30]). Ambiguities due to different prescriptions are comparable and in some cases much larger than relativistic corrections. In the 
application to electron scattering presented in sect. 4 we shall therefore omit off-shell corrections and compare results with different transition operators under the same kinematic conditions. Concluding remarks are given in sect. 5 .

\section{Gauge invariance and charge-current den- sity}

Let us consider a point particle with charge $e$, momentum $\vec{p}$ and Hamiltonian $H_{0}\left(=p^{0}\right)$. According to the usual prescription, the interaction Hamiltonian with the electromagnetic field is obtained by the minimal substitution

$$
p^{\mu} \rightarrow p^{\mu}-e A^{\mu}, \quad\left(p^{\mu}=\mathrm{i} \partial^{\mu}\right),
$$

where $A \equiv A^{\mu}=\left(A^{0}, A^{i}\right)$ is the electromagnetic field operator whose time dependence is understood. Greek indices run over 0, 1, 2, 3 and Latin ones over $1,2,3$, with a spatially negative metric tensor such that, e. g., $x^{i}=-x_{i}$, $x^{0}=x_{0}=t(\hbar=c=1)$.

The resulting Hamiltonian $H(A)$ is gauge invariant, i.e. the physical properties of the system remain unchanged under a gauge transformation of the field,

$$
A^{\mu} \rightarrow A^{\prime \mu}=A^{\mu}-\partial^{\mu} \lambda
$$

where $\lambda(\vec{x}, t)$ is an arbitrary function satisfying the D'Alembert equation. The requirement that the Schrödinger equation is conserved in form under the transformation of the Hamiltonian

$$
H(A) \rightarrow H^{\prime}(A)=H\left(A^{\prime}\right),
$$

when accompanied by the local phase transformation of the wavefunction

$$
\Psi(\vec{x}, t) \rightarrow \Psi^{\prime}(\vec{x}, t)=\mathrm{e}^{\mathrm{i} e \lambda(\vec{x}, t)} \Psi(\vec{x}, t),
$$

leads to the identity in $\lambda(t)$ and $\dot{\lambda}(t)$,

$$
H\left(A^{\prime}\right)+e \dot{\lambda}(t)=\mathrm{e}^{\mathrm{i} e \lambda(t)} H(A) \mathrm{e}^{-\mathrm{i} e \lambda(t)},
$$

where $\lambda(t)$ and $\dot{\lambda}(t)$ are operators multiplying the wavefunction by $\lambda(\vec{x}, t)$ and $\partial_{t} \lambda(\vec{x}, t)$, respectively. 
The interaction Hamiltonian with the electromagnetic field,

$$
H_{\mathrm{em}}(A) \equiv H(A)-H_{0}
$$

can usefully be expanded in a Taylor series of functional derivatives. Within the first quantization formalism it is the expectation value of an operator $O(A)$ given as a functional of the four components $A^{\mu}(\vec{x}, t)$ to be considered as functions of $\vec{x}$ depending on the parameter $t$. The functional derivatives of the operator $O(A)$ are then defined weakly by the relation

$$
\left\langle\Psi\left|\frac{\delta O(A)}{\delta A^{\mu}(\vec{x}, t)}\right| \Psi\right\rangle=\frac{\delta}{\delta A^{\mu}(\vec{x}, t)}\langle\Psi|O(A)| \Psi\rangle
$$

for all $|\Psi\rangle$ belonging to the domain of $O(A)$. The operators $A^{\nu}$ themselves can be considered as functionals of the functions $A^{\mu}(\vec{x}, t)$, i.e.

$$
\left\langle\Psi\left|A^{\nu}\right| \Psi\right\rangle=\int \mathrm{d} \vec{x} \int \mathrm{d} \vec{y}\langle\Psi \mid \vec{y}\rangle A^{\nu}(\vec{x}, t) \delta(\vec{x}-\vec{y})\langle\vec{y} \mid \Psi\rangle .
$$

Then, from the definition (11) one has

$$
\frac{\delta A^{\nu}}{\delta A^{\mu}(\vec{x}, t)}=\delta_{\mu \nu} \rho(\vec{x})
$$

where $\rho(\vec{x})$ is the density operator defined by

$$
\langle\vec{y}|\rho(\vec{x})| \Psi\rangle=\delta(\vec{x}-\vec{y})\langle\vec{y} \mid \Psi\rangle \text {. }
$$

The density operator is also useful to express the operators $\lambda(t)$ and $\dot{\lambda}(t)$. Using eq. (14) one readily obtains

$$
\lambda(t)=\int \mathrm{d} \vec{x} \rho(\vec{x}) \lambda(\vec{x}, t), \quad \dot{\lambda}(t)=\int \mathrm{d} \vec{x} \rho(\vec{x}) \partial_{t} \lambda(\vec{x}, t) .
$$

Therefore, $\lambda(t)$ and $\dot{\lambda}(t)$ can be considered as functionals of $\lambda(\vec{x}, t)$ and $\partial_{t} \lambda(\vec{x}, t)$, respectively, and

$$
\frac{\delta \lambda(t)}{\delta \lambda(\vec{x}, t)}=\frac{\delta \dot{\lambda}(t)}{\delta \partial_{t} \lambda(\vec{x}, t)}=\rho(\vec{x})
$$


As a consequence, both sides of eq. (9) are functionals of $\lambda(\vec{x}, t)$ and $\partial_{t} \lambda(\vec{x}, t)$. Using eq. (16) and the law for the derivative of a composed functional, the corresponding functional derivatives (evaluated at $\lambda(\vec{x}, t)=0$ and $\left.\partial_{t} \lambda(\vec{x}, t)=0\right)$ give (see appendix)

$$
\begin{gathered}
\frac{\partial}{\partial x_{i}}\left(\frac{\delta H(A)}{\delta A^{i}(\vec{x}, t)}\right)=-\mathrm{i} e[H(A), \rho(\vec{x})], \\
\frac{\delta H(A)}{\delta A^{0}(\vec{x}, t)}=e \rho(\vec{x}) .
\end{gathered}
$$

Thus, the quantities

$$
J_{\mu}(A, \vec{x}) \equiv \frac{\delta H(A)}{\delta A^{\mu}(\vec{x}, t)}
$$

satisfy the continuity equation

$$
\frac{\partial}{\partial x_{i}} J_{i}(A, \vec{x})=-\mathrm{i}\left[H(A), J_{0}(A, \vec{x})\right]
$$

and are interpreted as the components of the charge-current density operator. From eqs. (17) and (18) it appears that $J_{i}(A, \vec{x})$ depend on $t$ only through their functional dependence on $A^{\mu}$ and $J_{0}(A, \vec{x})$ does not depend on $A^{\mu}$.

Let us expand $H(A)$ into the functional Taylor series

$$
H(A)=H_{0}+\int \mathrm{d} \vec{x} j_{\mu}(\vec{x}) A^{\mu}(\vec{x}, t)+\frac{1}{2} \int \mathrm{d} \vec{x} \int \mathrm{d} \vec{y} A^{\mu}(\vec{x}, t) b_{\mu \nu}(\vec{x}, \vec{y}) A^{\nu}(\vec{y}, t)+\ldots,
$$

where

$$
j_{\mu}(\vec{x})=\left.\frac{\delta H(A)}{\delta A^{\mu}(\vec{x}, t)}\right|_{A=0}, \quad b_{\mu \nu}(\vec{x}, \vec{y})=\left.\frac{\delta^{2} H(A)}{\delta A^{\mu}(\vec{x}, t) \delta A^{\nu}(\vec{y}, t)}\right|_{A=0} .
$$

These operators do not depend on $t$ (see eqs. (27) and (28) below). In addition, due to the independence of the order of functional differentiation the following symmetry property holds

$$
b_{\mu \nu}(\vec{x}, \vec{y})=b_{\nu \mu}(\vec{y}, \vec{x}) .
$$


Now let us relate $j_{\mu}(\vec{x})$ and $b_{\mu \nu}(\vec{x}, \vec{y})$ to $J_{\mu}(A, \vec{x})$. Since $H(A)$ linearly depends on $A^{0}$, one has

$$
\begin{gathered}
j_{0}(\vec{x})=J_{0}(A, \vec{x})=e \rho(\vec{x}), \\
b_{0 \nu}(\vec{x}, \vec{y})=b_{\mu 0}(\vec{x}, \vec{y})=0 .
\end{gathered}
$$

Using eq. (21) one has

$$
J_{i}(A, \vec{x})=j_{i}(\vec{x})+\int \mathrm{d} \vec{y} b_{i j}(\vec{x}, \vec{y}) A^{j}(\vec{y}, t)+\ldots
$$

Thus, $j_{i}(\vec{x})$ is the first-order expression of the current-density operator.

Inserting eqs. (21) and (26) into eq. (17), which identically holds in $A^{\mu}$, one obtains

$$
\begin{gathered}
\frac{\partial}{\partial x_{i}} j_{i}(\vec{x})=-\mathrm{i} e\left[H_{0}, \rho(\vec{x})\right], \\
\frac{\partial}{\partial x_{i}} b_{i j}(\vec{x}, \vec{y})=-\mathrm{i} e\left[j_{j}(\vec{y}), \rho(\vec{x})\right] .
\end{gathered}
$$

\section{Electromagnetic interaction in semi-relativ- istic theories}

Ignoring spin degrees of freedom for the time being, let us consider the following semi-relativistic Hamiltonian

$$
H_{0}=\sqrt[+]{\vec{p}^{2}+m^{2}}+V,
$$

where the self-adjoint operator $V$ is energy and momentum independent. Under the action of the electromagnetic field

$$
A^{\mu}(x)=\epsilon^{\mu} \mathrm{e}^{\mathrm{i}\left(\vec{q} \cdot \vec{x}-q_{0} t\right)},
$$

the minimal substitution gives

$$
H(A)=\sqrt[+]{(\vec{p}-e \vec{A})^{2}+m^{2}}+e A_{0}+V .
$$

Since the operators $A^{\mu}$ are bounded, the operator $(\vec{p}-e \vec{A})^{2}$ is self-adjoint. Hence, also the positive square root in eq. (31) is a well-defined self-adjoint operator. 
Due to eq. (21), the interaction Hamiltonian with the electromagnetic field is written (up to second order) as

$$
H_{\mathrm{em}}(A)=H_{\mathrm{em}}^{(1)}(A)+H_{\mathrm{em}}^{(2)}(A),
$$

where

$$
\begin{gathered}
H_{\mathrm{em}}^{(1)}(A)=\int \mathrm{d} \vec{x} j_{\mu}(\vec{x}) A^{\mu}(\vec{x}, t), \\
H_{\mathrm{em}}^{(2)}(A)=\frac{1}{2} \int \mathrm{d} \vec{x} \int \mathrm{d} \vec{y} A^{i}(\vec{x}, t) b_{i j}(\vec{x}, \vec{y}) A^{j}(\vec{y}, t) .
\end{gathered}
$$

\subsection{Expression of $H_{\mathrm{em}}^{(1)}(A)$}

The charge density operator $j_{0}$ is already known from eq. (24). The firstorder current density operator can be written as

$$
j_{i}(\vec{x})=\left.\frac{\delta R(A)}{\delta A^{i}(\vec{x}, t)}\right|_{A=0},
$$

where

$$
R(A)=\sqrt[+]{(\vec{p}-e \vec{A})^{2}+m^{2}} .
$$

The r.h.s. of eq. (35) can be obtained from the easily calculable functional derivative of $[R(A)]^{2}$. One has

$$
\left\{R(A), \frac{\delta R(A)}{\delta A^{i}(\vec{x}, t)}\right\}_{A=0}=\left.\frac{\delta[R(A)]^{2}}{\delta A^{i}(\vec{x}, t)}\right|_{A=0}=e\left\{p_{i}, \rho(\vec{x})\right\} .
$$

The anticommutators in eq. (37) can be solved in momentum representation, where from eq. (14) one has

$$
\left\langle\vec{k}^{\prime}|\rho(\vec{x})| \vec{k}\right\rangle=(2 \pi)^{-3} \mathrm{e}^{\mathrm{i}\left(\vec{k}-\vec{k}^{\prime}\right) \cdot \vec{x}} .
$$

Thus eqs. (35) and (37) give

$$
\left\langle\vec{k}^{\prime}\left|j_{\mu}(\vec{x})\right| \vec{k}\right\rangle=e \frac{k_{\mu}+k_{\mu}^{\prime}}{k_{0}+k_{0}^{\prime}}(2 \pi)^{-3} \mathrm{e}^{\mathrm{i}\left(\vec{k}-\vec{k}^{\prime}\right) \cdot \vec{x}},
$$

where

$$
k_{0} \equiv E_{\vec{k}}=\sqrt[+]{\vec{k}^{2}+m^{2}}, \quad k_{0}^{\prime} \equiv E_{\vec{k}^{\prime}}=\sqrt[+]{\vec{k}^{\prime 2}+m^{2}} .
$$


One easily checks that the continuity equation (27) is satisfied.

From eqs. (21) and (39) the interaction with the electromagnetic field is (up to first order in $e$ )

$$
\left\langle\vec{k}^{\prime}\left|H_{\mathrm{em}}^{(1)}(A)\right| \vec{k}\right\rangle=e \frac{\epsilon^{\mu}\left(k_{\mu}+k_{\mu}^{\prime}\right)}{k_{0}+k_{0}^{\prime}} \delta\left(\vec{k}+\vec{q}-\vec{k}^{\prime}\right) \mathrm{e}^{-\mathrm{i} q_{0} t} .
$$

In terms of operators eq. (41) reads

$$
H_{\mathrm{em}}^{(1)}(A)=e\left(\epsilon_{0}-\frac{\vec{\epsilon} \cdot(2 \vec{p}-\vec{q})}{\sqrt[+]{\vec{p}^{2}+m^{2}}+\sqrt[+]{(\vec{p}-\vec{q})^{2}+m^{2}}}\right) \tilde{\rho}(\vec{q}) \mathrm{e}^{-\mathrm{i} q_{0} t},
$$

where

$$
\tilde{\rho}(\vec{q})=\int \mathrm{d} \vec{x} \rho(\vec{x}) \mathrm{e}^{\mathrm{i} \vec{q} \cdot \vec{x}}
$$

\subsection{Expression of $H_{\mathrm{em}}^{(2)}(A)$}

For the operators $b_{i j}(\vec{x}, \vec{y})$ the following relation

$$
b_{i j}(\vec{x}, \vec{y})=\left.\frac{\delta^{2} R(A)}{\delta A^{i}(\vec{x}, t) \delta A^{j}(\vec{y}, t)}\right|_{A=0}
$$

can be derived from

$$
\left\{R(A), \frac{\delta^{2} R(A)}{\delta A^{i}(\vec{x}, t) \delta A^{j}(\vec{y}, t)}\right\}=\frac{\delta^{2}[R(A)]^{2}}{\delta A^{i}(\vec{x}, t) \delta A^{j}(\vec{y}, t)}-\left\{\frac{\delta R(A)}{\delta A^{i}(\vec{x}, t)}, \frac{\delta R(A)}{\delta A^{j}(\vec{y}, t)}\right\} .
$$

Using eq. (35) and calculating the second-order derivative of $[R(A)]^{2}$ one has

$$
\left\{R(A), \frac{\delta^{2} R(A)}{\delta A^{i}(\vec{x}, t) \delta A^{j}(\vec{y}, t)}\right\}=2 e^{2} \delta_{i j} \rho(\vec{x}) \rho(\vec{y})-\left\{j_{i}(\vec{x}), j_{j}(\vec{y})\right\},
$$

so that

$$
\left\langle\vec{k}^{\prime}\left|b_{i j}(\vec{x}, \vec{y})\right| \vec{k}\right\rangle=\frac{1}{k_{0}+k_{0}^{\prime}}\left\langle\vec{k}^{\prime}\left|\left[2 \delta_{i j} j_{0}(\vec{x}) j_{0}(\vec{y})-\left\{j_{i}(\vec{x}), j_{j}(\vec{y})\right\}\right]\right| \vec{k}\right\rangle .
$$

Hence, the second-order correction of the electromagentic interaction is

$$
\begin{gathered}
\left\langle\vec{k}^{\prime}\left|H_{\mathrm{em}}^{(2)}(A)\right| \vec{k}\right\rangle=\frac{e^{2}}{E_{\vec{k}}+E_{\vec{k}^{\prime}}}\left[\vec{\epsilon} \cdot \vec{\epsilon}+\frac{(\vec{\epsilon} \cdot \vec{q})^{2}-4\left(\vec{\epsilon} \cdot\left(\vec{k}^{\prime}-\vec{q}\right)\right)^{2}}{\left(E_{\vec{k}^{\prime}}+E_{\vec{k}^{\prime}-\vec{q}}\right)\left(E_{\vec{k}}+E_{\vec{k}^{\prime}-\vec{q}}\right)}\right] \\
\times \delta\left(\vec{k}+2 \vec{q}-\vec{k}^{\prime}\right) \mathrm{e}^{-2 \mathrm{i} q_{0} t} .
\end{gathered}
$$




\subsection{Spin contribution}

To treat a particle with spin $\frac{1}{2}$, one endows the wavefunctions with the matrix structure related to the Pauli matrices $\sigma_{i}$ and writes the Hamiltonian $H_{0}$ of eq. (29) in the equivalent form

$$
H_{0}=\sqrt[+]{(\vec{\sigma} \cdot \vec{p})^{2}+m^{2}}+V
$$

Therefore, the minimal substitution gives

$$
H(A)=\sqrt[+]{(\vec{p}-e \vec{A})^{2}-e \vec{\sigma} \cdot \vec{B}+m^{2}}+e A_{0}+V
$$

where

$$
\vec{B}=\vec{\nabla} \times \vec{A}
$$

The first-order contribution of the spin to the charge-current density,

$$
j_{\mu}^{S}(\vec{x})=\left.\frac{\delta H^{S}(A)}{\delta A^{\mu}(\vec{x}, t)}\right|_{A=0},
$$

where

$$
H^{S}(A)=H(A)-\sqrt[+]{(\vec{p}-e \vec{A})^{2}+m^{2}}-e A_{0}-V,
$$

can be calculated applying the method of sect. 3.1 to each single term in $H^{S}(A)$ and using the relations

$$
\begin{gathered}
\frac{\delta \vec{\sigma} \cdot \vec{B}}{\delta A^{i}(\vec{x}, t)}=\mathrm{i} \frac{\delta}{\delta A^{i}(\vec{x}, t)}[(\vec{\sigma} \times \vec{p}) \cdot \vec{A}-\vec{A} \cdot(\vec{\sigma} \times \vec{p})]=-\mathrm{i}\left[(\vec{\sigma} \times \vec{p})_{i}, \rho(\vec{x})\right], \\
\frac{\delta \vec{\sigma} \cdot \vec{B}}{\delta A^{0}(\vec{x}, t)}=0 .
\end{gathered}
$$

One obtains

$$
\begin{gathered}
\left\langle\vec{k}^{\prime}, s^{\prime}\left|\vec{j}^{S}(\vec{x})\right| \vec{k}, s\right\rangle=\frac{\mathrm{i} e}{k_{0}+k_{0}^{\prime}}\left\langle s^{\prime}\left|\vec{\sigma} \times\left(\vec{k}^{\prime}-\vec{k}\right)\right| s\right\rangle(2 \pi)^{-3} e^{\mathrm{i}\left(\vec{k}-\vec{k}^{\prime}\right) \cdot \vec{x}}, \\
\left\langle\vec{k}^{\prime}, s^{\prime}\left|\vec{j}_{0}(\vec{x})\right| \vec{k}, s\right\rangle=0 .
\end{gathered}
$$

The corresponding contribution to the first-order interaction Hamiltonian is

$$
\left\langle\vec{k}^{\prime}, s^{\prime}\left|H_{\mathrm{em}}^{S(1)}(A)\right| \vec{k}, s\right\rangle=-\frac{e}{k_{0}+k_{0}^{\prime}}\left\langle\vec{k}^{\prime}, s^{\prime}|\vec{\sigma} \cdot \vec{B}| \vec{k}, s\right\rangle .
$$




\section{Application to quasi-elastic electron scat- tering}

In order to compare the semi-relativistic charge-current density operator obtained in sect. 3 with other operators existing in the literature we consider quasi-elastic electron scattering. The advantage of this process is that it is almost independent of any details of the nuclear structure. In fact, neglecting final-state interactions the coincidence $\left(e, e^{\prime} p\right)$ cross section for unpolarized electrons is simply proportional to the (off-shell) electron-proton cross section $\sigma_{\text {ep }}$ [1],

$$
\frac{\mathrm{d}^{3} \sigma}{\mathrm{d} E^{\prime} \mathrm{d} \Omega \mathrm{d} \Omega_{\mathrm{p}}}=K \sigma_{\mathrm{ep}} S(\vec{p}, E)
$$

where $K$ is a kinematical factor and $S(\vec{p}, E)$ is the spectral function for knocking out a proton of momentum $\vec{p}$ and energy $E$. For a coplanar kinematics,

$$
\sigma_{\mathrm{ep}}=\sigma_{\mathrm{M}}\left(\rho_{00} g_{00}+\rho_{11} g_{11}+\rho_{01} g_{01}+\rho_{1-1} g_{1-1}\right),
$$

where $\sigma_{\mathrm{M}}$ is the Mott cross section describing elastic Coulomb scattering by a point-like particle. The coefficients $\rho_{\lambda \lambda^{\prime}}$ only depend on the electron kinematic variables and are well known from quantum electrodynamics [1]. Thus, the four structure functions $g_{\lambda \lambda^{\prime}}$ (with $\lambda=0$ for the longitudinal polarization and $\lambda= \pm 1$ for the two transverse polarizations of the exchanged photon) are the relevant quantities to study the effects due to the different charge-current density operators. In particular, $g_{00}$ and $g_{11}$ contain the pure

contribution of the charge and current density, respectively, while interference terms produce $g_{01}$ and $g_{1-1}$.

The $\sigma_{\text {ep }}$ most commonly used was proposed by de Forest 28], who made a detailed comparison of the existing cross sections in different kinematical situations. The nuclear current of eq. (2) produces the cross section $\sigma_{\mathrm{cc} 1}$, while the one of eq. (1) gives $\sigma_{\mathrm{cc} 2}$. On shell the two cross sections are equal, but even off shell they are very close to each other. However, $\sigma_{\mathrm{cc} 1}$, which is widely used, is simpler and using on-shell kinematics the separated contributions to $\sigma_{\mathrm{ep}}$ are given by

$$
g_{00}=\frac{\left(E_{\vec{p}}+E_{\vec{p}^{\prime}}\right)^{2}}{4 E_{\vec{p}} E_{\vec{p}^{\prime}}}\left(F_{1}^{2}+\frac{Q^{2}}{4 M^{2}} \kappa^{2} F_{2}^{2}\right)-\frac{1}{4 E_{\vec{p}} E_{\vec{p}}}|\vec{q}|^{2}\left(F_{1}+\kappa F_{2}\right)^{2},
$$




$$
\begin{aligned}
g_{11} & =\frac{\left|\vec{p}^{\prime}\right|^{2} \sin ^{2} \gamma}{E_{\vec{p}} E_{\vec{p}^{\prime}}}\left(F_{1}^{2}+\frac{Q^{2}}{4 M^{2}} \kappa^{2} F_{2}^{2}\right)+\frac{Q^{2}}{2 E_{\vec{p}} E_{\vec{p}^{\prime}}}\left(F_{1}+\kappa F_{2}\right)^{2}, \\
g_{01} & =-\frac{\sqrt{2}\left(E_{\vec{p}}+E_{\vec{p}^{\prime}}\right)}{E_{\vec{p}} E_{\vec{p}^{\prime}}}\left|\vec{p}^{\prime}\right| \sin \gamma\left(F_{1}^{2}+\frac{Q^{2}}{4 M^{2}} \kappa^{2} F_{2}^{2}\right), \\
g_{1-1} & =-\frac{\left|\vec{p}^{\prime}\right|^{2} \sin ^{2} \gamma}{E_{\vec{p}} E_{\vec{p}^{\prime}}}\left(F_{1}^{2}+\frac{Q^{2}}{4 M^{2}} \kappa^{2} F_{2}^{2}\right) .
\end{aligned}
$$

where $\gamma$ is the angle between $\vec{q}$ and $\vec{p}^{\prime}$,

$$
E_{\vec{p}}=\sqrt{|\vec{p}|^{2}+M^{2}}, \quad E_{\vec{p}^{\prime}}=\sqrt{\left|\overrightarrow{p^{\prime}}\right|^{2}+M^{2}}
$$

are the initial and final proton energy, respectively, and

$$
\omega=E_{\vec{p}^{\prime}}-E_{\vec{p}}
$$

is the energy transfer with $\vec{p}^{\prime}=\vec{p}+\vec{q}$.

The same structure functions calculated with the non-relativistic nucleon charge-current operator and truncated to order $M^{-2}$ are given by

$$
\begin{aligned}
g_{00} & =F_{1}^{2}-F_{1}\left(F_{1}+2 \kappa F_{2}\right) \frac{1}{4 M^{2}} Q^{2} \\
g_{11} & =F_{1}^{2} \frac{1}{M^{2}}\left|\vec{p}^{\prime}\right|^{2} \sin ^{2} \gamma+\left(F_{1}+\kappa F_{2}\right)^{2} \frac{1}{2 M^{2}}|\vec{q}|^{2} \\
g_{01} & =-2 \sqrt{2} F_{1}^{2} \frac{1}{M}\left|\vec{p}^{\prime}\right| \sin \gamma \\
g_{1-1} & =-F_{1}^{2} \frac{1}{M^{2}}\left|\vec{p}^{\prime}\right|^{2} \sin ^{2} \gamma .
\end{aligned}
$$

Higher-order corrections are obtained following the approach of ref. [6].

The corresponding expressions in the semi-relativistic case are:

$$
g_{00}=F_{1}^{2}
$$




$$
\begin{aligned}
g_{11} & =F_{1}^{2} \frac{4}{\left(E_{\vec{p}}+E_{\vec{p}^{\prime}}\right)^{2}}\left|\vec{p}^{\prime}\right|^{2} \sin ^{2} \gamma+\left(F_{1}+\kappa F_{2}\right)^{2} \frac{2}{\left(E_{\vec{p}}+E_{\vec{p}^{\prime}}\right)^{2}}|\vec{q}|^{2}, \\
g_{01} & =-2 \sqrt{2} F_{1}^{2} \frac{2}{E_{\vec{p}}+E_{\vec{p}^{\prime}}}\left|\vec{p}^{\prime}\right| \sin \gamma, \\
g_{1-1} & =-F_{1}^{2} \frac{4}{\left(E_{\vec{p}}+E_{\vec{p}^{\prime}}\right)^{2}}\left|\vec{p}^{\prime}\right|^{2} \sin ^{2} \gamma .
\end{aligned}
$$

In the semi-relativistic case the charge and current densities include form factors in order to describe an extended particle and, correspondingly, the contribution of the anomalous magnetic moment has been added to the spin current.

There are two differences between the semi-relativistic and non-relativistic structure functions. First, the Darwin-Foldy (DF) term appearing in the non-relativistic $g_{00}$ cannot be reproduced in the semi-relativistic case, because only the spatial components of the charge-current density operator are affected when going from the non-relativistic kinetic energy to the semirelativistic one in eq. (29), while the time component is simply given by the charge density. In fact, the DF term arises in the non-relativistic reduction of the Dirac equation which has negative-energy solutions, whereas the semirelativistic Hamiltonian does not have such solutions. Second, apart from the DF term, the semi-relativistic structure functions are obtained from the non-relativistic ones by substituting the mass $M$ with $\frac{1}{2}\left(E_{\vec{p}}+E_{\vec{p}^{\prime}}\right)$ so that they coincide in the low-energy limit, $E \approx M$. In this limit they also become equal to the structure functions of the relativistic $\sigma_{\mathrm{cc} 1}$ apart from the $Q^{2}$-dependent terms involving the anomalous magnetic moment in the convective current and the charge density. Precisely these terms are responsible for the observed deviations from a non-relativistic description of the quasifree knockout in the standard kinematics explored up to now. Of course, larger deviations between the relativistic and non-relativistic structure functions are expected for increasing energy and momentum transfers.

In order to study the behaviour of the current given by the different approaches one has to focus on the pure transverse structure function, $g_{11}$. Assuming the result $g_{11}(\mathrm{cc} 1)$ obtained within the cross section $\sigma_{\mathrm{cc} 1}$ as a reference, the percentage deviation from this result calculated within the other 
approaches,

$$
\Delta=\frac{g_{11}-g_{11}(\mathrm{cc} 1)}{g_{11}(\mathrm{cc} 1)} \times 100
$$

will be considered as an indication of how far one is from the correct relativistic result.

In fig. 1 results are shown in parallel kinematics, i.e. when $\vec{q}$ and $\vec{p}$ are aligned, for three values of $p^{\prime}=0.5,0.75$ and $1 \mathrm{GeV}$. The socalled perpendicular kinematics is used in figs. 2 and 3, i.e. $q$ and $p^{\prime}$ are fixed and the angle $\gamma$ between $\vec{q}$ and $\vec{p}^{\prime}$ is varying. In fig. $2, p^{\prime}=0.5 \mathrm{GeV}$, while in fig. 3, $p^{\prime}=1 \mathrm{GeV}$. The corresponding results for $p^{\prime}=0.75 \mathrm{GeV}$ can be interpolated from those in figs. 2 and 3. For $\gamma=0^{\circ}$ one recovers the result in parallel kinematics at the corresponding $q$ value.

As already known from the analysis of ref. [6], the non-relativistic result (to order $M^{-2}$ ) and that corrected to order $M^{-3}$ are always of opposite sign with respect to the result corrected to order $M^{-4}$. The latter is within a few percent from the relativistic case for $p^{\prime}=0.5 \mathrm{GeV}$ both in parallel and perpendicular kinematics. However, for larger momenta the deviation rapidly deteriorates, in agreement with ref. [5], where it was stressed that the non-

relativistic FW reduction becomes meaningless for values of $p / M$ larger than 0.5 , with $p$ a typical momentum. On the contrary, the semi-relativistic result is comparable with the non-relativistic one at low energy and soon merges into the relativistic one for increasing $q$ and $p^{\prime}$.

\section{Concluding remarks}

Starting from a single-particle semi-relativistic Hamiltonian, where only the kinetic part has a relativistic shape, the corresponding charge-current density and two-photon operators have been obtained within a suitable functional derivative formalism. Such operators satisfy gauge invariance by construction and fully describe the behaviour of a point particle undergoing an external electromagnetic interaction as long as the effects of negative-energy states can be neglected.

In particular, the matrix elements of the current density operator between free-particle states coincide with the non-relativistic expressions of the usual convection and spin currents only in the low-energy limit when the total energy of the particle can simply be approximated by its mass. On the 
other hand, once eliminated the presence of negative-energy states ab initio, no corrections to the non-relativistic charge operator are possible in the present semi-relativistic approach. Nonetheless, the operators obtained here are valid for energies up to the threshold of particle-antiparticle production, thus substantially increasing the range of applicability with respect to any non-relativistic reduction of the electromagnetic interaction. In fact, it is well known that non-relativistic charge-current density operators, even corrected up to fourth order in $M^{-1}$, do not apply for values of $p / M$ larger than 0.5 , with $p$ a typical momentum. On the contrary, a comparison between results obtained with the semi-relativistic current density operator and a fully relativistic operator presented in the case of electron-proton scattering shows a more and more satisfactory agreement for increasing values of the ejectile momentum and/or momentum transfer.

Therefore, whenever the impulse approximation applies, the proposed semi-relativistic operators could also be used to describe the electromagnetic interaction of a many-body system at intermediate energies.

This work has been performed in part under the contract ERB FMRXCT-96-0008 within the frame of the Training and Mobility of Researchers Programme of the Commission of the European Union. 


\section{Appendix}

In this appendix the derivation of eqs. (17) and (18) is given.

$H\left(A^{\prime}\right)$ is a functional of the functions $A^{\prime \prime}(\vec{y}, t)=A^{i}(\vec{y}, t)-\partial \lambda(\vec{y}, t) / \partial y_{i}$ and $A^{\prime 0}(\vec{y}, t)=A^{0}(\vec{y}, t)-\partial \lambda(\vec{y}, t) / \partial t$ which in turn are functionals (dependent on the parameter $\vec{y})$ of the functions $\lambda(\vec{x}, t)$ and $\partial \lambda(\vec{x}, t) / \partial t$ :

$$
\begin{aligned}
& A^{\prime i}(\vec{y}, t)=A^{i}(\vec{y}, t)+\int \mathrm{d} \vec{x} \frac{\partial \delta(\vec{x}-\vec{y})}{\partial x_{i}} \lambda(\vec{x}, t), \\
& A^{\prime 0}(\vec{y}, t)=A^{0}(\vec{y}, t)-\int \mathrm{d} \vec{x} \delta(\vec{x}-\vec{y}) \frac{\partial \lambda(\vec{x}, t)}{\partial t} .
\end{aligned}
$$

By the law of differentiation of a composed functional one obtains

$$
\begin{aligned}
\frac{\delta H\left(A^{\prime}\right)}{\delta \lambda(\vec{x}, t)} & =\int \mathrm{d} \vec{y} \frac{\delta H\left(A^{\prime}\right)}{\delta A^{\prime}(\vec{y}, t)} \frac{\delta A^{\prime i}(\vec{y}, t)}{\delta \lambda(\vec{x}, t)}, \\
\frac{\delta H\left(A^{\prime}\right)}{\delta\left(\partial_{t} \lambda(\vec{x}, t)\right)} & =\int \mathrm{d} \vec{y} \frac{\delta H\left(A^{\prime}\right)}{\delta A^{\prime 0}(\vec{y}, t)} \frac{\delta A^{\prime 0}(\vec{y}, t)}{\delta\left(\partial_{t} \lambda(\vec{x}, t)\right)},
\end{aligned}
$$

where, due eqs. (67) and (68),

$$
\begin{gathered}
\frac{\delta A^{\prime i}(\vec{y}, t)}{\delta \lambda(\vec{x}, t)}=\frac{\partial \delta(\vec{x}-\vec{y})}{\partial x_{i}}, \\
\frac{\delta A^{\prime 0}(\vec{y}, t)}{\delta\left(\partial_{t} \lambda(\vec{x}, t)\right)}=-\delta(\vec{x}-\vec{y}) .
\end{gathered}
$$

Therefore,

$$
\begin{gathered}
\left.\frac{\delta H\left(A^{\prime}\right)}{\delta \lambda(\vec{x}, t)}\right|_{\lambda=0}=\int \mathrm{d} \vec{y} \frac{\delta H(A)}{\delta A^{i}(\vec{y}, t)} \frac{\partial \delta(\vec{x}-\vec{y})}{\partial x_{i}}=\frac{\partial}{\partial x_{i}} \frac{\delta H(A)}{\delta A^{i}(\vec{x}, t)} \\
\left.\frac{\delta H\left(A^{\prime}\right)}{\delta\left(\partial_{t} \lambda(\vec{x}, t)\right)}\right|_{\lambda=0}=-\int \mathrm{d} \vec{y} \frac{\delta H(A)}{\delta A^{0}(\vec{y}, t)} \delta(\vec{x}-\vec{y})=-\frac{\delta H(A)}{\delta A^{0}(\vec{y}, t)}
\end{gathered}
$$

One is now left with the evaluation of the functional derivative of $\mathrm{e}^{\mathrm{i} e \lambda(t)}$ which is a function of the functional $\lambda(t)$ of $\lambda(\vec{x}, t)$. Due to the first eq. (16) one has:

$$
\frac{\delta \mathrm{e}^{\mathrm{i} e \lambda(t)}}{\delta \lambda(\vec{x}, t)}=\left.\mathrm{i} e \mathrm{e}^{\mathrm{i} e \lambda(t)} \rho(\vec{x}) \Longrightarrow \frac{\delta \mathrm{e}^{\mathrm{i} e \lambda(t)}}{\delta \lambda(\vec{x}, t)}\right|_{\lambda=0}=\mathrm{i} e \rho(\vec{x}) .
$$

Using eqs. (73), (74), (75) and the second eq. (16) one obtains eqs. (17) and (18). 


\section{References}

[1] S. Boffi, C. Giusti, F.D. Pacati and M. Radici, Electromagnetic Response of Atomic Nuclei, Oxford University Press, Oxford, 1996.

[2] H.W. Fearing, G.I. Poulis and S. Scherer, Nucl. Phys. A570 (1994) 657.

[3] F.E. Close and Zhenping Li, Phys. Rev. D42 (1990) 2194.

[4] L.L. Foldy and S.A. Wouthuysen, Phys. Rev. 78 (1950) 29.

[5] K.W. McVoy and L. Van Hove, Phys. Rev. 125 (1962) 1034.

[6] C. Giusti and F.D. Pacati, Nucl. Phys. A336 (1980) 427.

[7] W.M. Alberico, T.W. Donnelly and A. Molinari, Nucl. Phys. A512 (1990) 541.

[8] B.D. Serot and J.D. Walecka, Adv. Nucl. Phys. 16 (1986) 1.

[9] B. Bakamjian and L.H. Thomas, Phys. Rev. 92 (1953) 1300.

[10] L.L. Foldy, Phys. Rev. 122 (1961) 275.

[11] R.A. Krajcik and L.L. Foldy, Phys. Rev. D10 (1974) 1777.

[12] J.L. Friar, Phys. Rev. C12 (1975) 695.

[13] J. Carlson, J. Kogut and V.R. Pandharipande, Phys. Rev. D27 (1983) 233.

[14] S. Godfrey and N. Isgur, Phys. Rev. D32 (1985) 189.

[15] S. Capstick and N. Isgur, Phys. Rev. D34 (1986) 2809.

[16] M. Warns, H. Schröder, W. Pfeil and H. Rollnik, Z. Phys. C45 (1990) 613.

[17] L.Ya. Glozman, Z. Papp, W. Plessas, K. Varga and R.F. Wagenbrunn, LANL nucl-th/9705011

[18] W. Glöckle, T.-S.H. Lee and F. Coester, Phys. Rev. C33 (1986) 709. 
[19] J. Carlson, V.R. Pandharipande and R. Schiavilla, Phys. Rev. C47 (1993) 484.

[20] J.L. Forest, V.R. Pandharipande and J.L. Friar, Phys. Rev. C52 (1995) 568.

[21] J.L. Forest, V.R. Pandharipande, J. Carlson and R. Schiavilla, Phys. Rev. C52 (1995) 576.

[22] S. Capstick, Phys. Rev. D46 (1992) 2864.

[23] S. Capstick, Phys. Rev. D47 (1993) 1994.

[24] M. Warns, H. Schröder, W. Pfeil and H. Rollnik, Z. Phys. C45 (1990) 627.

[25] M. Warns, H. Schröder, W. Pfeil and H. Rollnik, Phys. Rev. D42 (1990) 2215 .

[26] H. Arenövel, in New Vistas in Electro-Nuclear Physics, ed. by E.L. Tomusiak, H.S. Caplan and E.T. Dressler, Plenum, 1986, pp. 251-292.

[27] S. Frullani and J. Mougey, Adv. Nucl. Phys. 14 (1984) 1.

[28] T. De Forest, Jr., Nucl. Phys. A392 (1983) 232.

[29] H.W.L. Naus, S.J. Pollock, J.H. Koch and U. Oelfke, Nucl. Phys. A509 (1990) 717 .

[30] H.W.L. Naus, J.H. Koch and J.L. Friar, Phys. Rev. C41 (1990) 2852. 


\section{Figure captions}

Fig. 1. The percentage deviation $\Delta$ of the pure transverse structure function

$g_{11}$ from the value obtained within the cross section $\sigma_{\mathrm{cc} 1}$, calculated within the semi-relativistic approach and the non-relativistic ones corrected through second $\left(1 / m^{2}\right)$, third $\left(1 / m^{3}\right)$ and fourth order $\left(1 / m^{4}\right)$. At the indicated values of the momentum of the proton ejected under parallel kinematic conditions, i.e. $\vec{p}$ parallel to the momentum transfer $\vec{q}, \Delta$ is plotted as a function of $q$.

Fig. 2. The percentage deviation $\Delta$ of the pure transverse structure function $g_{11}$ from the value obtained within the cross section $\sigma_{\mathrm{cc} 1}$, calculated within the semi-relativistic approach and the non-relativistic ones corrected through second $\left(1 / m^{2}\right)$, third $\left(1 / m^{3}\right)$ and fourth order $\left(1 / m^{4}\right)$. At the indicated values of the momentum transfer $q, \Delta$ is plotted for $p^{\prime}=0.5 \mathrm{GeV}$ as a function of the angle $\gamma$ between $\vec{q}$ and the the momentum $\vec{p}^{\prime}$ of the ejected proton.

Fig. 3. The same as in fig. 2 for $p^{\prime}=1 \mathrm{GeV}$. 

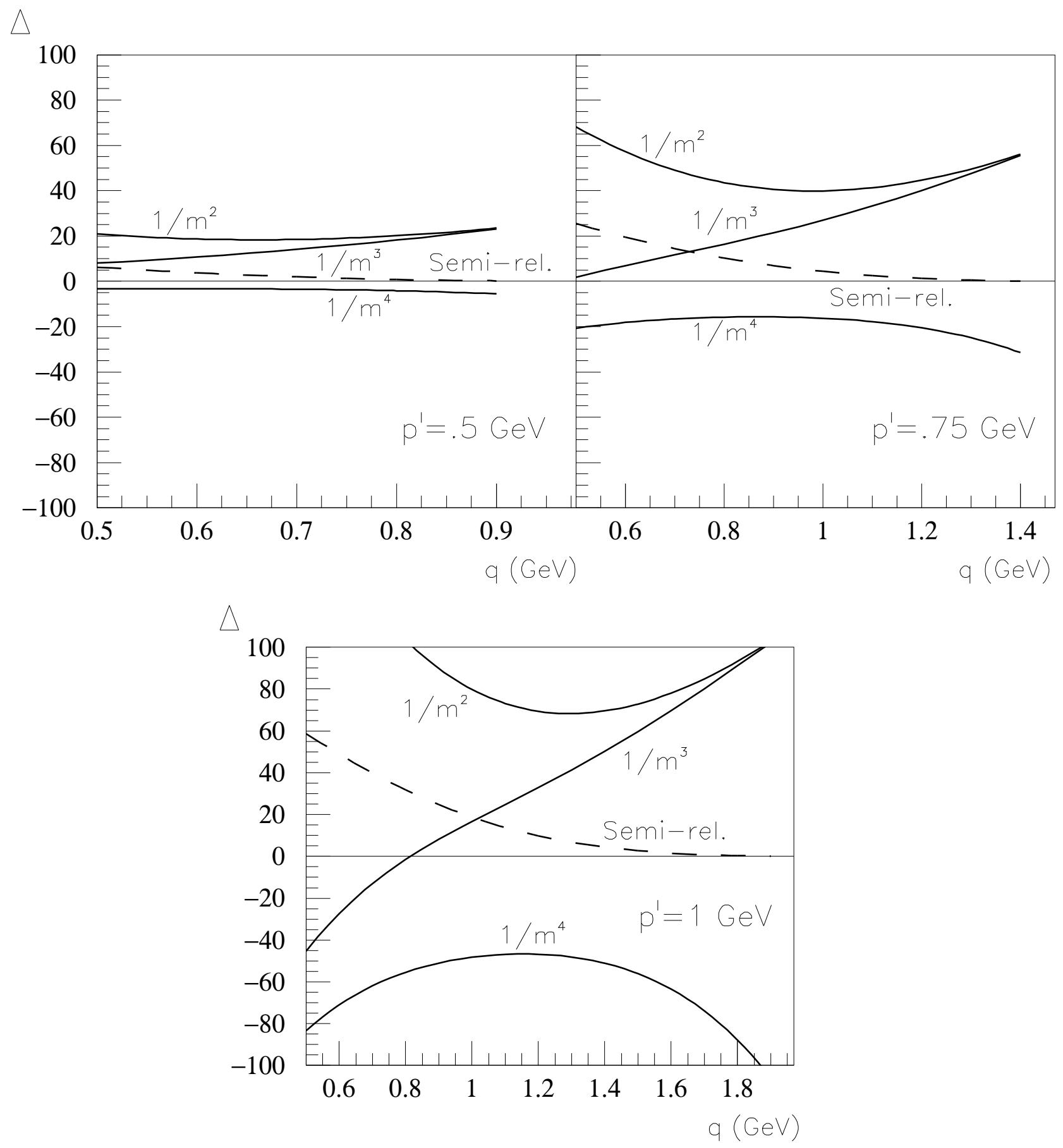

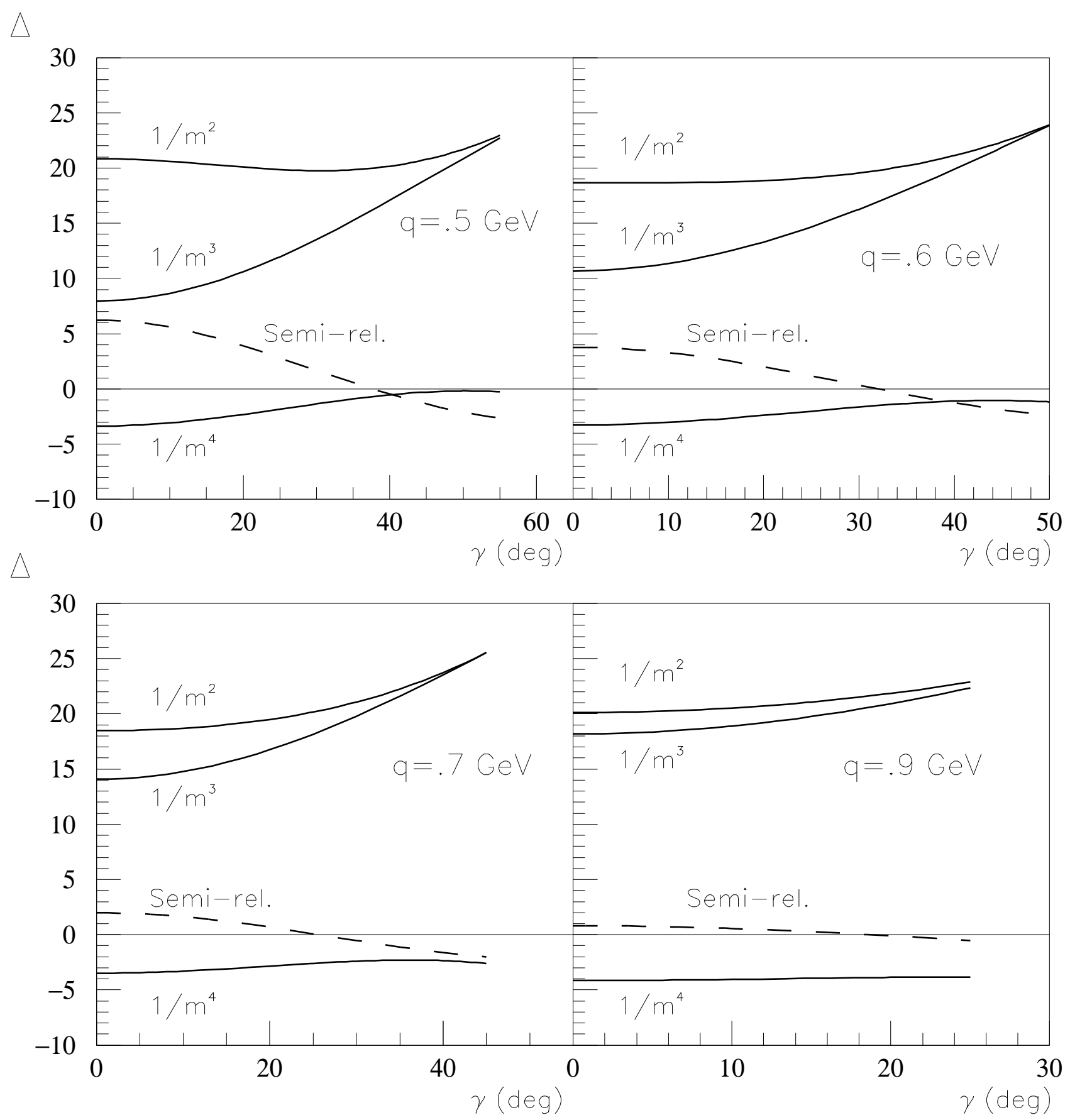

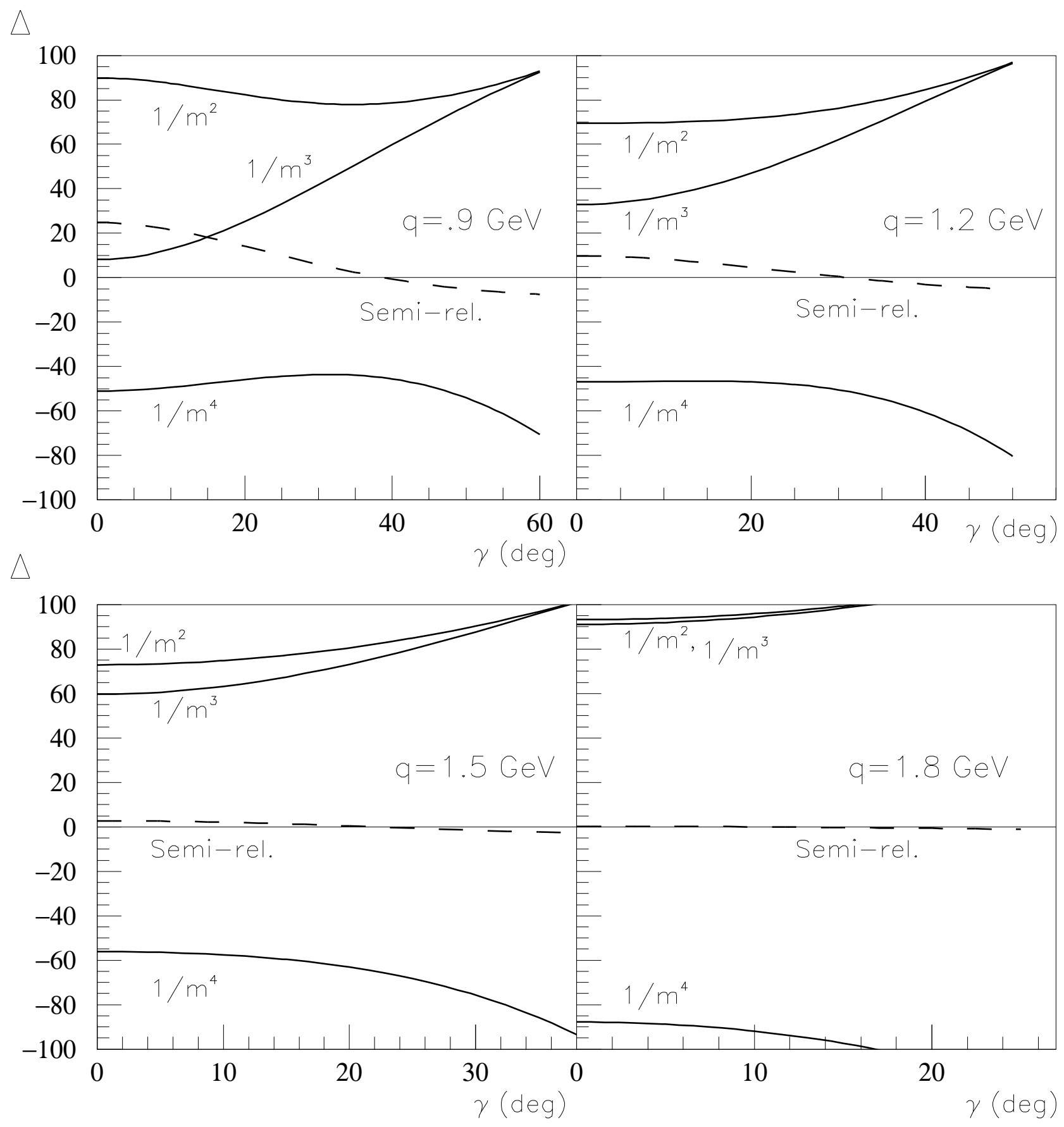